\title{
An Optimal Split-Plot Design for Performing a Mixture-Process Experiment
}

\author{
Gladys Gakenia Njoroge ${ }^{1}$, Jemimah Ayuma Simbauni ${ }^{2}$, Joseph Arap Koske ${ }^{3}$ \\ ${ }^{1}$ Department of Physical Sciences, Chuka University, Chuka, Kenya \\ ${ }^{2}$ Department of Zoological Sciences, Kenyatta University, Nairobi, Kenya \\ ${ }^{3}$ Department of Mathematics and Computer Science, Moi University, Eldoret, Kenya
}

Email address:

gg.njoroge@gmail.com (G. G. Njoroge), jsimbauni@yahoo.com (J. A. Simbauni), koske4@yahoo.co.uk (J. A. Koske)

\section{To cite this article:}

Gladys Gakenia Njoroge, Jemimah Ayuma Simbauni, Joseph Arap Koske. An Optimal Split-Plot Design for Performing a Mixture-Process Experiment. Science Journal of Applied Mathematics and Statistics. Vol. 5, No. 1, 2017, pp. 15-23.doi: 10.11648/j.sjams.20170501.13

Received: October 30, 2016; Accepted: November 21, 2016; Published: January 18, 2017

\begin{abstract}
In many mixture-process experiments, restricted randomization occurs and split-plot designs are commonly employed to handle these situations. The objective of this study was to obtain an optimal split-plot design for performing a mixture-process experiment. A split-plot design composed of a combination of a simplex centroid design of three mixture components and a $2^{2}$ factorial design for the process factors was assumed. Two alternative arrangements of design points in a split-plot design were compared. Design-Expert ${ }^{\circledR}$ version 10 software was used to construct I-and D-optimal split-plot designs. This study employed A-, D-, and E- optimality criteria to compare the efficiency of the constructed designs and fraction of design space plots were used to evaluate the prediction properties of the two designs. The arrangement, where there were more subplots than whole-plots was found to be more efficient and to give more precise parameter estimates in terms of A-, D- and E-optimality criteria. The I-optimal split-plot design was preferred since it had the capacity for better prediction properties and precision in the measurement of the coefficients. We thus recommend the employment of split-plot designs in experiments involving mixture formulations to measure the interaction effects of both the mixture components and the processing conditions. In cases where precision of the results is more desirable on the mixtures as well as where the mixture blends are more than the sets of process conditions, we recommend that the mixture experiment be set up at each of the points of a factorial design. In situations where the interest is on prediction aspects of the system, we recommend the I-optimal split-plot design to be employed since it has low prediction variance in much of the design space and also gives reasonably precise parameter estimates.
\end{abstract}

Keywords: Optimality, Split-Plot Design, Efficiency, Mixture Components, Process Variables

\section{Introduction}

In many practical situations, the response for the mixture may not just depend on the mixture components but also on experimental conditions that are referred to as the process variables. Mixture-process variable experiments are common in many fields such as food, chemical, pharmaceutical and processing industries. The process variables are not part of the mixture components but their levels when changed could affect the blending properties of the components [4]. Many of the mixture-process experiments are usually designed by combining a mixture design for the mixture ingredients and a process design for the process factors. The choice of the combination of the mixture design and the process variable design depend on the purpose of the design. Kowalski, Cornell and Vining [10] proposed a Split-Plot Design (SPD) for mixture-process variable experiments. The basic split-plot design involves assigning the levels of one factor to main plots (referred to as whole-plots) which can be arranged as a Completely Randomised Design (CRD), Randomised Complete Block Design (RCBD) or a Latin Square Design (LSD) and then assigning the levels of the second factor to sub-plots within each main plot. The mixture-process variables in the SPD structure have two levels of randomization leading to two types of errors; the whole plot and the subplot errors. Montgomery [13] recommended splitplot design as the way to deal with restricted randomization. 
A split-plot experiment can be carried out by randomly selecting a combination of levels of the process variables and running all mixture blends at these levels. Then, another combination of the process variable levels is randomly chosen and all mixture blends run at this combination. This process is repeated until all combination of levels of the process variables is performed. In such a case, the process variables are the whole-plot factors of the experiment, while the mixture components are the sub-plot factors. On the other hand, a mixture blend can be randomly chosen and run over all combinations of the process factors. This process is repeated until all the mixture blends are run over all the levels of combinations of the process factors. In this later case, the mixture components are the whole-plot factors and the process variables are the sub-plot factors.

Optimal designs are usually constructed using statistical packages and design optimality criteria such as A-optimality, D-optimality, E-optimality, G-optimality and I-optimality are employed. The criteria are chosen depending on the experimenter's interest. To evaluate and compare different designs, a number of methods have been proposed in literature. The concept of rotatability for the design was proposed by [2]. Rotatability requires constant prediction variance at all points that are the same distance from the design centre. When the interest is in the prediction variance at a specific location in the design space, design efficiency becomes a good measure for comparing and evaluating designs, [1]. The optimal criteria that focus on prediction variance at specific location in the design space include the Q-optimality, the V-optimality, Ioptimality and the G-optimality, [1]. When the experimenter's interest is on obtaining precise parameter estimates, Doptimality as advocated by [12], is useful. A-and E-optimality criteria are also used for precise parameter estimates. Since the prediction variance keeps changing at different points in the design, prediction capability of a design should be based on the overall distribution of the prediction variance over the whole design space rather than using the estimate of a single point. This led to the introduction of the graphical techniques to give a measure of the overall distribution of the prediction variance. The three dimensional variance dispersion graphs (VDG) for mixture-process variables was introduced first by [6] and later proposed by [8]. Fraction of design space (FDS) plots involve calculation of prediction variance throughout the design space and then the fraction of the design space that is less than or equal to a given prediction value is determined. FDS plots for SPDs were developed by [11]. The graphical assessment of experimental designs using FDS plots in $\mathrm{R}$ statistical environment was demonstrated by [14]. Good prediction properties are essential for optimization as [13] posits and Ioptimal designs provide this. A number of commercial statistical packages such as Design-Expert or JMP employ Dand I-optimality criteria for constructing the optimal designs. When a single design is to be employed in an experiment and the experimenter has interest in both precise parameter estimates and good prediction capability, then a design with good level of both properties would be preferred.

This study focussed on obtaining an optimal split-plot design that could be used to perform a mixture-process experiment that would give both precise parameter estimates and have good prediction capabilities. To do this, we first compared different split-plot design arrangements using A-, Dand E-efficiencies to obtain the most suitable design arrangement for a mixture-process experiment. This was followed by the construction of a D- and an I-optimal split-plot designs using Design-Expert software and employing the most suitable design arrangement obtained in the first step. The precision of the parameter estimates of the D- and I-optimal designs was measured and compared using A-, D- and Eoptimal values and efficiencies respectively.The prediction capability of the two SPDs was measured using FDS plots.

\section{Material and Methods}

\subsection{Design Arrangement Comparison}

A split-plot design composed of a combination of a simplex centroid design of three mixture components and a $2^{2}$ factorial design for two process factors was assumed for illustration purposes. The split-plot design consisted of 28 treatment combinations. The three mixture components were denoted as $x_{1}, x_{2}, x_{3}$ and set up in a simplex-centroid design with the following seven blends;

$$
\left(x_{1}, x_{2}, x_{3}\right)=(1,0,0),(0,1,0),(0,0,1),\left(\frac{1}{2}, \frac{1}{2}, 0\right),\left(\frac{1}{2}, 0 \frac{1}{2}\right),\left(0, \frac{1}{2}, \frac{1}{2}\right),\left(\frac{1}{3}, \frac{1}{3}, \frac{1}{3}\right)
$$

The two process factors coded as $z_{1}$ and $z_{2}$ had two levels each which were denoted as $z_{1}= \pm 1$ and $z_{2}= \pm 1$, laid out as:

$$
\left(z_{1}, z_{2}\right)^{\prime}=(-1,-1),(-1,1),(1,-1),(1,1)
$$

There are two alternative arrangements for the split-plot design which involve having either: the seven mixture blends in equation (1) set up at each of the four points in equation (2) of the factorial arrangement, as shown in fig. 1 (a) or the factorial design set up at each of the seven points of the simplex centroid design as shown in fig. 1 (b) below:

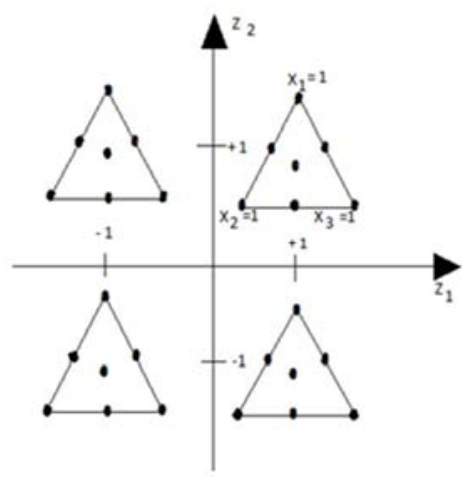

(a) 


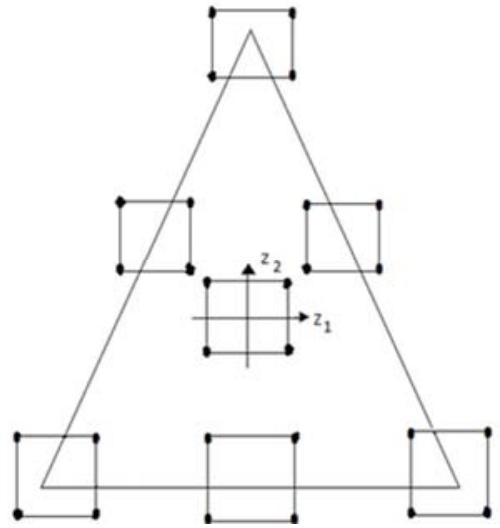

(b)

Figure 1. Arrangement for the 28 split-plot design points.

The corresponding model for each of the design arrangements (a) or (b) in fig. 1 was:

Model 1, for design arrangement (a)

$$
\begin{gathered}
\eta(x, z)=\delta_{1}^{0} x_{1}+\delta_{2}^{0} x_{2}+\delta_{3}^{0} x_{3}+\delta_{12}^{0} x_{1} x_{2}+\delta_{13}^{0} x_{1} x_{3}+ \\
\delta_{23}^{0} x_{2} x_{3}+\delta_{123}^{0} x_{1} x_{2} x_{3}+\delta_{1}^{1} z_{1} x_{1}+\delta_{2}^{1} z_{1} x_{2}+\delta_{3}^{1} z_{1} x_{3}+ \\
\delta_{12}^{1} z_{1} x_{1} x_{2}+\delta_{13}^{1} z_{1} x_{1} x_{3}+\delta_{23}^{1} z_{1} x_{2} x_{3}+\delta_{123}^{1} z_{1} x_{1} x_{2} x_{3}+ \\
\delta_{1}^{2} z_{2} x_{1}+\delta_{2}^{2} z_{2} x_{2}+\delta_{3}^{2} z_{2} x_{3}+\delta_{12}^{2} z_{2} x_{1} x_{2}+\delta_{13}^{2} z_{2} x_{1} x_{3}+ \\
\delta_{23}^{2} z_{2} x_{2} x_{3}+\delta_{123}^{2} z_{2} x_{1} x_{2} x_{3}+\delta_{1}^{12} z_{1} z_{2} x_{1}+\delta_{2}^{12} z_{1} z_{2} x_{2}+ \\
\delta_{3}^{12} z_{1} z_{2} x_{3}+\delta_{12}^{12} z_{1} z_{2} x_{1} x_{2}+\delta_{13}^{12} z_{1} z_{2} x_{1} x_{3}+\delta_{23}^{12} z_{1} z_{2} x_{2} x_{3}+ \\
\delta_{123}^{12} z_{1} z_{2} x_{1} x_{2} x_{3}
\end{gathered}
$$

Model 2 for design arrangement (b);

$\eta(x, z)=\delta_{1}^{0} x_{1}+\delta_{1}^{1} z_{1} x_{1}+\delta_{1}^{2} z_{2} x_{1}+\delta_{1}^{12} z_{1} z_{2} x_{1}+\delta_{2}^{0} x_{2}+$ $\delta_{2}^{1} z_{1} x_{2}+\delta_{2}^{2} z_{2} x_{2}+\delta_{2}^{12} z_{1} z_{2} x_{2}+\delta_{3}^{0} x_{3}+\delta_{3}^{1} z_{1} x_{3}+\delta_{3}^{2} z_{2} x_{2}+$ $\delta_{3}^{12} z_{1} z_{2} x_{3}+\delta_{12}^{0} x_{1} x_{2}+\delta_{12}^{1} z_{1} x_{1} x_{2}+\delta_{12}^{2} z_{2} x_{1} x_{2}+$ $\delta_{12}^{12} z_{1} z_{2} x_{1} x_{2}+\delta_{13}^{0} x_{1} x_{3}+\delta_{13}^{1} z_{1} x_{1} x_{3}+\delta_{13}^{2} z_{2} x_{1} x_{3}+$ $\delta_{13}^{12} z_{1} z_{2} x_{1} x_{3}+\delta_{23}^{0} x_{2} x_{3}+\delta_{23}^{1} z_{1} x_{2} x_{3}+\delta_{23}^{2} z_{2} x_{2} x_{3}+$ $\delta_{23}^{12} z_{1} z_{2} x_{2} x_{3}+\delta_{123}^{0} x_{1} x_{2} x_{3}+\delta_{123}^{1} z_{1} x_{1} x_{2} x_{3}+$$$
\delta_{123}^{2} z_{2} x_{1} x_{2} x_{3}+\delta_{123}^{12} z_{1} z_{2} x_{1} x_{2} x_{3}
$$

To decide on which of the two design arrangements 1(a) or (b) was more efficient, some design comparison strategies were employed on the two to find out which one was more efficient as explained in section (2.2) below.

\subsection{Optimal Split-Plot Design Construction}

The Design-Expert ${ }^{\circledR}$ version10 software [5] was used to construct an I-optimal completely randomized combined mixture-process design using the point-exchange algorithm. The software was also used to construct a D-optimal splitplot design. The two designs had the same number of design points. The I-optimal completely randomized mixture process design was then arranged to fit a split-plot format. The two split-plot designs were then compared.

\subsection{Evaluation and Comparison of Designs}

A design that has small number of runs minimizes the cost of performing the experiment, but this should not be at the expense of compromising other important aspects of a good design. A design with low prediction variance throughout the design space and with high capability of estimating the corresponding model parameters is more preferred. The methods used in evaluating and comparing designs include the use of design efficiency, from the alphabetic criteria and/or use of graphical techniques. This study employed A-, E-, and D- criteria to compare the efficiency of the constructed designs in giving precise parameter estimates and also the fraction of design space plots to evaluate their prediction capabilities.

The statistical model corresponding to a split-plot design, with $n$ observations arranged in $w$ whole-plots each of size $n_{j},(j=1,2, \ldots, w)$, is a linear mixed model of the form

$$
y=X \beta+Z \gamma+\varepsilon
$$

where $\boldsymbol{y}$ is the $(n \times 1)$ vector of observed responses, $\boldsymbol{X}$ is the $(n \times p)$ design matrix containing the settings of the explanatory variables in each experimental run, with $p$ being the number of model parameters, $\boldsymbol{\beta}$ is the associated $(p \times 1)$ vector of model parameters, $\boldsymbol{Z}$ is a matrix of zeroes and ones assigning the observations to the whole-plots, $\gamma$ is the $w$ dimensional vector of whole-plot effects while $\boldsymbol{\varepsilon}$ is the $(n \times$ $1)$ vector of random errors. Model (5) contains two random components, one for the whole-plot $(\boldsymbol{\gamma})$ and the other for the sub-plot $(\varepsilon)$, [9]. It is usually assumed that

$$
\begin{array}{r}
E(\boldsymbol{\varepsilon})=\boldsymbol{o}_{\boldsymbol{n}} \text { and } \operatorname{cov}(\boldsymbol{\varepsilon})=\sigma_{\varepsilon}^{2} \boldsymbol{I}_{n}, \\
E(\boldsymbol{\gamma})=\boldsymbol{o}_{\boldsymbol{w}} \text { and } \operatorname{cov}(\boldsymbol{\gamma})=\sigma_{\gamma}^{2} \boldsymbol{I}_{w} \\
\text { And } \operatorname{cov}(\gamma, \varepsilon)=\boldsymbol{o}_{\boldsymbol{w} \times \boldsymbol{n}}
\end{array}
$$

This can be presented as:

$\boldsymbol{\varepsilon} \sim N\left(0_{n}, \sigma_{\varepsilon}^{2} I_{n}\right), \gamma \sim N\left(0_{w}, \sigma_{\gamma}^{2} I_{w}\right)$ and $\operatorname{cov}(\varepsilon, \gamma)=0_{n \times w}$. Under these assumptions the variance-covariance matrix of the observations, $\operatorname{var}(y)$ is written as $\boldsymbol{V}=\sigma_{\varepsilon}^{2} \boldsymbol{I}_{n}+\sigma_{\gamma}^{2} \mathbf{Z Z}^{\prime}$ which can be re-written as

$$
\boldsymbol{V}=\sigma_{\varepsilon}^{2}\left(\boldsymbol{I}_{n}+\eta \mathbf{Z} \mathbf{Z}^{\prime}\right)
$$

where $\eta=\sigma_{\gamma}^{2} / \sigma_{\varepsilon}^{2}$ is a measure of the extent to which the observations within the same whole plot are correlated and is referred to as variance component ratio, [3]. For the purpose of design construction, it is the ratio of both variance components, $\eta$, that matters but not their absolute magnitude, [9]. Let the entries of $\boldsymbol{y}$ be arranged per whole-plot, then

$$
\begin{aligned}
& \boldsymbol{V}=\left[\begin{array}{cccc}
V_{1} & 0 & \cdots & 0 \\
\vdots & \ddots & \vdots \\
0 & 0 & \cdots & V_{w}
\end{array}\right] \\
& \boldsymbol{Z Z}^{\prime}=\left[\begin{array}{ccccc}
1_{n_{1}} 1_{n_{1}}^{\prime} & 0 & 0 & 0 \\
0 & 1_{n_{2}} 1_{n_{2}}^{\prime} & 0 & 0 \\
\vdots & \vdots & \ddots & & \vdots \\
0 & 0 & 0 & & 1_{w} 1_{w}^{\prime}
\end{array}\right]
\end{aligned}
$$

The length of each $1_{n_{j}}$ is $n_{j}$, the number of sub-plot runs within the whole-plot. The information matrix on the unknown fixed model parameters $\beta$ is given by

$$
\boldsymbol{M}=\boldsymbol{X}^{T} \boldsymbol{V}^{-1} \boldsymbol{X}
$$


The variance components $\sigma_{\gamma}^{2}$ and $\sigma_{\varepsilon}^{2}$ and matrix $\mathrm{V}$ are unknown and hence need to be estimated as shown in equation (12) below.

$$
\widehat{V}=\hat{\sigma}_{\varepsilon}^{2}\left(I_{n}+\eta Z Z^{\prime}\right)
$$

When different values of $\eta$ are substituted in equation (13), estimates of $V$ can be obtained and values of $M$ calculated as:

$$
M=X^{T} \widehat{V}^{-1} X
$$

In cases where the random error terms as well as the whole-plot effects are normally distributed, the maximum likelihood estimate of $\beta$ is the generalized least squares (GLS) estimate and not the ordinary least squares (OLS) estimate, [9]. Consequently, the estimators for the unknown model parameters $\beta$ are:

$$
\hat{\beta}=\left(X^{T} V^{-1} X\right)^{-1} X^{T} V^{-1} y
$$

and the variance-covariance matrix of the estimators is given by

$$
\operatorname{var}(\hat{\beta})=\left(X^{T} V^{-1} X\right)^{-1}
$$

Once the estimates of the variance components are obtained, they are substituted in equation (13) whose value is in turn substituted in formula (14) to get the so-called feasible GLS estimator, [9].

$$
\hat{\beta}=\left(\boldsymbol{X}^{T} \widehat{\boldsymbol{V}}^{-1} \boldsymbol{X}\right)^{-1} \boldsymbol{X}^{T} \widehat{\boldsymbol{V}}^{-1} y
$$

As a result, the variance-covariance matrix in equation (15) could be approximated by

$$
\operatorname{var}(\hat{\beta})=\left(X^{T} \hat{V}^{-1} X\right)^{-1}
$$

\subsubsection{A-Optimality Criterion}

The A-optimality criterion is based on a measure of the minimum average variance of the parameter estimates. Thus, it seeks to minimize the trace of the inverse of the information matrix. For a split-plot design, it is given by

$$
\min \operatorname{tr}\left(X^{T} V^{-1} X\right)^{-1}
$$

On comparing two designs $\xi_{1}$ and $\xi_{2}$, the design with a lower value from formula (18) is considered more efficient and is preferred in terms of A-optimality criterion.

\subsubsection{D-Optimality Criterion}

The D-optimal designs are commonly used for precise parameter estimation. Its aim is to maximize the determinant of the information matrix, thus in turn minimizing the generalized variance. For this criterion, a design that makes the information matrix large in some sense is desirable.

The D-efficiency is calculated as

$$
\left[\left|M_{1}\right| /\left|M_{2}\right|\right]^{1 / p}
$$

where $M_{1}$ and $M_{2}$ are the information matrices for design $\xi_{1}$ and $\xi_{2}$ respectively, while $p$ is the number of model parameters. Design $\xi_{1}$ is better than $\xi_{2}$ in terms of D- optimality criterion if a D-efficiency calculated from formula (19) is larger than 1.

\subsubsection{E-optimality Criterion}

The aim of the E-optimality criterion is to minimize the maximum variance of all the possible normalized linear combinations of parameter estimates. This implies maximizing the minimum Eigenvalue of the information matrix. It is given by the maximum Eigen value of min $\operatorname{tr}\left(X^{T} V^{-1} X\right)^{-1}$ or minimum Eigen value of $\left(X^{T} V^{-1} X\right)$. E-efficiency is given by

$$
E\left(\xi_{1}\right)=\frac{\lambda_{\min }\left(M\left(\xi_{1}\right)\right)}{\lambda_{\min }\left(M\left(\xi_{2}\right)\right)}
$$

If $\lambda_{\min }\left(M\left(\xi_{1}\right)\right)>\lambda_{\min }\left(M\left(\xi_{2}\right)\right)$, then design $\xi_{1}$ is more efficient than design $\xi_{2}$ and is preferred in terms of Eoptimality criterion.

\subsubsection{The FDS Plots}

Fraction of design space plots are based on the argument that for any accurate prediction over the entire design space $\chi$, the fraction of the volume of $\chi$ that is associated with various values of the split-plot design must be taken into account [7]. On the other hand, the variance dispersion graphs usually give equal weight to the scaled prediction variance (SPV) for all radii, $r$, despite the fact that the fraction of the volume of the design region represented by the hypersphere of each radius differs substantially. This may be interpreted to mean that a small SPV at a radius close to the origin may not give as a good prediction as a similar SPV value at a larger $r$, a drawback of the VDG. The FDS criterion however, expresses the volume of the set $\mathrm{R}$ containing all points in $\chi$ with SPV lower than $v$ as a proportion of the total design space volume, where $v$ is a fixed value of the $\operatorname{SPV} d\left(X, \xi_{n}\right)$ and $R=\{X \in$ $\left.\chi: d\left(X, \xi_{n}\right)<v\right\}$.

The FDS criterion is defined as $(v, \chi)=\phi^{-1} \int_{R} d x$, where $\Phi$ is the volume of $\chi$. For each specified SPV value $v$, the FDS plot graghs $v$ against FDS(v). FDS plots display the fraction of design space where SPV is less than or equal to $\mathrm{V}$, [3]. The FDS plots in this study were constructed using [5]. The purpose of the evaluation and comparison is to help identify the most competitive optimal design which can then be employed in performing an experiment appropriately.

\section{Results and Discussion}

\subsection{Split-Plot Design Arrangement Comparison}

The alphabetical criteria for optimality A, D and E were used to compare the two arrangements as explained in section (2.3). The $Z Z^{\prime}$ matrix in equation (3) for design arrangement 1(a) was

$$
Z Z^{\prime}=\left[\begin{array}{cccc}
1_{7} 1_{7}^{\prime} & \mathbf{0} & \mathbf{0} & \mathbf{0} \\
\mathbf{0} & 1_{7} 1_{7}^{\prime} & \mathbf{0} & \mathbf{0} \\
\mathbf{0} & \mathbf{0} & 1_{7} 1_{7}^{\prime} & \mathbf{0} \\
\mathbf{0} & \mathbf{0} & \mathbf{0} & 1_{7} 1_{7}^{\prime}
\end{array}\right]
$$

where, $1_{7} 1_{7}^{\prime}$ was a $7 \times 7$ matrix of ones and each 0 represents a 
$7 \times 7$ matrix of zeros.

For design arrangement $1(\mathrm{~b})$, the matrix $Z Z^{\prime}$ was of order $7 \times 7$

$$
Z Z^{\prime}=\left[\begin{array}{ccccc}
1_{4} 1_{4}^{\prime} & 0 & \cdots & 0 & 0 \\
0 & 1_{4} 1_{4}^{\prime} & \cdots & 0 & 0 \\
\vdots & \vdots & \ddots & \vdots & \vdots \\
0 & 0 & \cdots & 1_{4} 1_{4}^{\prime} & 0 \\
0 & 0 & \cdots & 0 & 1_{4} 1_{4}^{\prime}
\end{array}\right]
$$

where, $1_{4}$ was a $4 \times 4$ matrix of ones and each 0 represents a $4 \times 4$ matrix of zeros.

The identity matrix $I_{n}$ in equation (12) was the $I_{28}$ of order $28 \times 28$ since there was a total of 28 design points in each of the arrangements. Values of variance component ratio $(\eta)$ in equation (12) used in this section for comparison of design arrangement 1 (a) and (b) were $0.1,1$ and 10 .

The variance-covariance matrix $\mathrm{V}$ was obtained as in the equation (12) and was then substituted in equation (13) to

obtain the information matrix $\mathrm{M}$ using the $\mathrm{R}$ statistical software. The $\mathrm{X}$ matrix in equation (13) was a $28 \times 28$ design matrix representing the fixed effects of combined mixture and process variables, ordered according to the design arrangement 1 (a) or (b).

Using $\boldsymbol{\eta}=\mathbf{1}$

A-optimal criterion

The $\operatorname{tr}\left\{\left[M\left(\xi_{a}\right)\right]^{-1}\right\}=1267.461$

$$
\begin{gathered}
\operatorname{tr}\left\{\left[M\left(\xi_{b}\right)\right]^{-1}\right\}=1979.947 \\
\operatorname{tr}\left\{\left[M\left(\xi_{a}\right)\right]^{-1}\right\}<\operatorname{tr}\left\{\left[M\left(\xi_{b}\right)\right]^{-1}\right\}
\end{gathered}
$$

Therefore, design arrangement 1(a) is more efficient than design arrangement 1(b) in terms of A-optimality and it is therefore preferred.

D-optimality criterion

$$
\begin{array}{ll}
\text { was then substituted in equation }(13) \text { to } & \left|M\left(\xi_{a}\right)\right|=2.1953 \mathrm{e}-13 \\
D_{\text {eff }}=\left\{\left[\left|M\left(\xi_{a}\right)\right|\right] /\left[\left|M\left(\xi_{b}\right)\right|\right]\right\}^{\frac{1}{28}}=[2.1953 \mathrm{e}-13 / 1.150969 \mathrm{e}-14]^{\frac{1}{28}}=1.11104
\end{array}
$$

Since the $D_{\text {eff }}$ value is greater than 1, then the design arrangement 1(a) is more efficient than design arrangement 1(b) in terms of D-optimality criterion.

E-optimality criterion

\begin{tabular}{|c|c|c|c|c|c|}
\hline \multirow{2}{*}{ Variance component ratio ( $\eta$ ) } & \multirow{2}{*}{ Optimality criterion } & \multicolumn{2}{|c|}{ Optimal-value } & \multirow{2}{*}{ Efficiency } & \multirow{2}{*}{ Comment } \\
\hline & & Design (a) & Design (b) & & \\
\hline \multirow[t]{3}{*}{$\eta=0.1$} & A-optimality & 1264.76 & 1336.009 & 0.947 & (a) preferred \\
\hline & D-optimality & $1.08 \mathrm{e}-10$ & $8.53 e-11$ & 0.96 & (a) preferred \\
\hline & E-optimality & 0.00333683 & 0.00287598 & 1.16 & (a) preferred \\
\hline \multirow[t]{3}{*}{$\eta=1$} & A-optimality & 1267.461 & 1979.947 & 0.64 & (a) preferred \\
\hline & D-optimality & $2.20 \mathrm{e}-13$ & $1.15 \mathrm{e}-14$ & 1.11104 & (a) preferred \\
\hline & E-optimality & 0.003336827 & 0.0012778 & 2.61 & (a) preferred \\
\hline \multirow[t]{3}{*}{$\eta=10$} & A-optimality & 1294.467 & 8419.332 & 0.1537 & (a) preferred \\
\hline & D-optimality & $3.54 \mathrm{e}-17$ & $4.62 \mathrm{e}-21$ & 1.1426 & (a) preferred \\
\hline & E-optimality & 0.003336795 & 0.000194284 & 17.17 & (a) preferred \\
\hline
\end{tabular}

$$
\begin{gathered}
\lambda_{\text {min }} M\left(\xi_{a}\right)=0.003336827 \\
\lambda_{\text {min }} M\left(\xi_{b}\right)=0.001277800 \\
\lambda_{\text {min }} M\left(\xi_{a}\right)>\lambda_{\text {min }} M\left(\xi_{b}\right) \text { or } \frac{\lambda_{\min M\left(\xi_{a}\right)}}{\lambda_{\min } M\left(\xi_{b}\right)}=\frac{0.003336827}{0.001277800}=2.61>1
\end{gathered}
$$

Hence, design arrangement 1(a) is more efficient than design arrangement 1(b) and is therefore preferred in terms of Eoptimal criterion.

With $\eta=0.1$ and 10 , a table was created to summarize the findings as below:

Table 1. Optimal Values for Design Arrangement in fig. 1 Compared at Different Values of the Variance Component Ratio.

From the results in table 1, the most efficient split-plot design arrangement, in terms of E-, D- and E-optimality was found to be where the mixture blends were run at each of the points of the factorial design process conditions, (C. F. fig. 1(a)). This agreed with Cornell (2002) recommendation.

\subsection{Optimal Split-Plot Designs}

Design arrangement 1(a) was employed in the optimal split-plot design construction in this section. Two optimal split-plot designs: D-optimal and I-optimal were constructed using Design-Expert ${ }^{\circledR}$ version 10.The alphabetical optimality criteria A-, D- and E were employed to compare the two designs. FDS plots for the two designs were evaluated. The D-optimal design was coded as $\xi_{d}$ and the I-optimal design was coded as $\xi_{i}$. Each of the two designs had 38 points; 28 of which were model based, to estimate the 28 model parameters in equation (3), five additional points for testing the fitness of the model to the experimental data and another 
additional five points were replicate points to enable the calculation of the standard error. The main difference between the two optimal designs was in the position of the replicated points in the design space; the D-optimal design had the replicated points on the edges and vertices of the simplex as presented in table 2 while the I-optimal design had the replicates in the inside of the simplex mainly on the centroid as seen in table 3 .

Table 2. The D-optimal split-plot design $\left(\xi_{d}\right)$.

\begin{tabular}{|c|c|c|c|c|c|}
\hline Run order & $\mathbf{x}_{1}$ & $\mathbf{x}_{2}$ & $\mathbf{x}_{3}$ & $\mathbf{z}_{1}$ & $\mathbf{z}_{2}$ \\
\hline 1 & 0.3333 & 0.3333 & 0.3333 & 1 & 1 \\
\hline 2 & 0 & 0 & 1 & 1 & 1 \\
\hline 3 & 0.5 & 0 & 0.5 & 1 & 1 \\
\hline 4 & 0 & 0.5 & 0.5 & 1 & 1 \\
\hline 5 & 0 & 0 & 1 & 1 & 1 \\
\hline 6 & 0.5 & 0.5 & 0 & 1 & 1 \\
\hline 7 & 1 & 0 & 0 & 1 & 1 \\
\hline 8 & 0 & 1 & 0 & 1 & 1 \\
\hline 9 & 1 & 0 & 0 & 1 & 1 \\
\hline 10 & 0.5 & 0.5 & 0 & -1 & 1 \\
\hline 11 & 0 & 0.5 & 0.5 & -1 & 1 \\
\hline 12 & 0.3333 & 0.3333 & 0.3333 & -1 & 1 \\
\hline 13 & 1 & 0 & 0 & -1 & 1 \\
\hline 14 & 0 & 1 & 0 & -1 & 1 \\
\hline 15 & 0 & 0 & 1 & -1 & 1 \\
\hline 16 & 0.5 & 0 & 0.5 & -1 & 1 \\
\hline 17 & 1 & 0 & 0 & -1 & 1 \\
\hline 18 & 0 & 1 & 0 & 1 & -1 \\
\hline 19 & 0 & 0 & 1 & 1 & -1 \\
\hline 20 & 0.5 & 0 & 0.5 & 1 & -1 \\
\hline 21 & 1 & 0 & 0 & 1 & -1 \\
\hline 22 & 0 & 0.5 & 0.5 & 1 & -1 \\
\hline 23 & 1 & 0 & 0 & 1 & -1 \\
\hline 24 & 0.5 & 0.5 & 0 & 1 & -1 \\
\hline 25 & 0.3333 & 0.3333 & 0.3333 & 1 & -1 \\
\hline 26 & 0.5 & 0.5 & 0 & -1 & -1 \\
\hline 27 & 1 & 0 & 0 & -1 & -1 \\
\hline 28 & 0 & 1 & 0 & -1 & -1 \\
\hline 29 & 0.3333 & 0.3333 & 0.3333 & -1 & -1 \\
\hline 30 & 0 & 0 & 1 & -1 & -1 \\
\hline 31 & 0.5 & 0 & 0.5 & -1 & -1 \\
\hline 32 & 0 & 1 & 0 & -1 & -1 \\
\hline 33 & 0 & 0.5 & 0.5 & -1 & -1 \\
\hline 34 & 0.1667 & 0.6667 & 0.1667 & 1 & 0 \\
\hline 35 & 0.1667 & 0.6667 & 0.1667 & 0 & -1 \\
\hline 36 & 0 & 1 & 0 & 0 & 0 \\
\hline 37 & 0.1667 & 0.6667 & 0.1667 & -1 & 0 \\
\hline 38 & 0.1667 & 0.6667 & 0.1667 & 0 & 1 \\
\hline
\end{tabular}

Table 3. The I-optimal split-plot design $\left(\xi_{i}\right)$.

\begin{tabular}{|c|c|c|c|c|c|}
\hline Run order & $\mathbf{x}_{1}$ & $\mathbf{x}_{2}$ & $\mathbf{x}_{3}$ & $\mathrm{z}_{1}$ & $\mathbf{Z}_{2}$ \\
\hline 1 & 0.5 & 0.5 & 0 & -1 & -1 \\
\hline 2 & 0.5 & 0 & 0.5 & -1 & -1 \\
\hline 3 & 0 & 1 & 0 & -1 & -1 \\
\hline 4 & 0 & 0 & 1 & -1 & -1 \\
\hline 5 & 0.3333 & 0.3333 & 0.3333 & -1 & -1 \\
\hline 6 & 0 & 0.5 & 0.5 & -1 & -1 \\
\hline 7 & 0.3333 & 0.3333 & 0.3333 & -1 & -1 \\
\hline 8 & 0.3333 & 0.3333 & 0.3333 & -1 & -1 \\
\hline 9 & 1 & 0 & 0 & -1 & -1 \\
\hline 10 & 0.3333 & 0.3333 & 0.3333 & 1 & -1 \\
\hline 11 & 1 & 0 & 0 & 1 & -1 \\
\hline 12 & 0.3333 & 0.3333 & 0.3333 & 1 & -1 \\
\hline 13 & 0 & 1 & 0 & 1 & -1 \\
\hline 14 & 0.5 & 0 & 0.5 & 1 & -1 \\
\hline 15 & 0 & 0.5 & 0.5 & 1 & -1 \\
\hline 16 & 0.5 & 0.5 & 0 & 1 & -1 \\
\hline 17 & 0 & 0 & 1 & 1 & -1 \\
\hline 18 & 0 & 0 & 1 & -1 & 1 \\
\hline 19 & 0 & 0.5 & 0.5 & -1 & 1 \\
\hline 20 & 0.5 & 0 & 0.5 & -1 & 1 \\
\hline 21 & 0.3333 & 0.3333 & 0.3333 & -1 & 1 \\
\hline 22 & 1 & 0 & 0 & -1 & 1 \\
\hline 23 & 0.3333 & 0.3333 & 0.3333 & -1 & 1 \\
\hline 24 & 0.5 & 0.5 & 0 & -1 & 1 \\
\hline 25 & 0 & 1 & 0 & 1 & 1 \\
\hline 26 & 0.3333 & 0.3333 & 0.3333 & 1 & 1 \\
\hline 27 & 0.5 & 0.5 & 0 & 1 & 1 \\
\hline 28 & 1 & 0 & 0 & 1 & 1 \\
\hline 29 & 0 & 0.5 & 0.5 & 1 & 1 \\
\hline 30 & 0.5 & 0 & 0.5 & 1 & 1 \\
\hline 31 & 0.3333 & 0.3333 & 0.3333 & 1 & 1 \\
\hline 32 & 0 & 1 & 0 & 1 & 1 \\
\hline 33 & 0 & 0 & 1 & 1 & 1 \\
\hline 34 & 0 & 1 & 0 & 0 & -0.5 \\
\hline 35 & 0 & 0 & 1 & 0 & -0.5 \\
\hline 36 & 0.1667 & 0.6667 & 0.1667 & 1 & 0 \\
\hline 37 & 0.6667 & 0.1667 & 0.1667 & 0 & 0 \\
\hline 38 & 0.1667 & 0.6667 & 0.1667 & 0 & 1 \\
\hline
\end{tabular}

For the comparison purposes, only 33 design points from each of the designs $\xi_{d}$ and $\xi_{i}$ were included since there was no model testing that was involved at this stage. The included design points were the 28 model points and the 5 replicated points.

\subsection{Optimal Split-Plot Design Comparison}

Optimality tests were conducted following the steps in section (2.3).

The results of the optimality tests were as follows:

\subsubsection{A-optimality Criterion}

The $\operatorname{tr}\left\{\left[M\left(\xi_{d}\right)\right]^{-1}\right\}=1257.109$

$$
\begin{gathered}
\operatorname{tr}\left\{\left[M\left(\xi_{i}\right)\right]^{-1}\right\}=871.795 \\
\operatorname{tr}\left\{\left[M\left(\xi_{i}\right)\right]^{-1}\right\}<\operatorname{tr}\left\{\left[M\left(\xi_{d}\right)\right]^{-1}\right\}
\end{gathered}
$$

I-optimal split-plot design was more efficient in terms of A-optimality than the D-optimal split-plot design. 


\subsubsection{D-optimality Criterion}

$$
\begin{gathered}
\left|M\left(\xi_{d}\right)\right|=3.947083 \mathrm{e}-12 \\
\left|M\left(\xi_{i}\right)\right|=2.960312 \mathrm{e}-12 D_{\text {eff }=}\left\{\left[\left|M\left(\xi_{d}\right)\right|\right] /\left[\left|M\left(\xi_{i}\right)\right|\right]\right\}^{\frac{1}{33}}=[3.947083 \mathrm{e}-12 / 2.960312 \mathrm{e}-12]^{\frac{1}{33}}=1.008756
\end{gathered}
$$

This D-efficiency value was very close to 1 , implying that the efficiency of this I-optimal design in terms of Doptimality is acceptable.

\subsubsection{E-optimality Criterion}

$$
\begin{gathered}
\lambda_{\text {min }} M\left(\xi_{d}\right)=0.003348500 \\
\lambda_{\text {min }} M\left(\xi_{i}\right)=0.004775119 \\
\lambda_{\text {min }} M\left(\xi_{i}\right)>\lambda_{\text {min }} M\left(\xi_{d}\right)
\end{gathered}
$$

Hence, the I-optimal split-plot design was more efficient than the D-optimal split-plot design in terms of the Eoptimality criterion. Therefore, in terms of the three alphabetical optimality criteria used for the comparison of the two split-plot designs, the I-optimal design performed better. The evaluation was then extended to the FDS plots.

\subsubsection{The FDS Plots}

The FDS plot for the D-optimal split-plot design was as below:

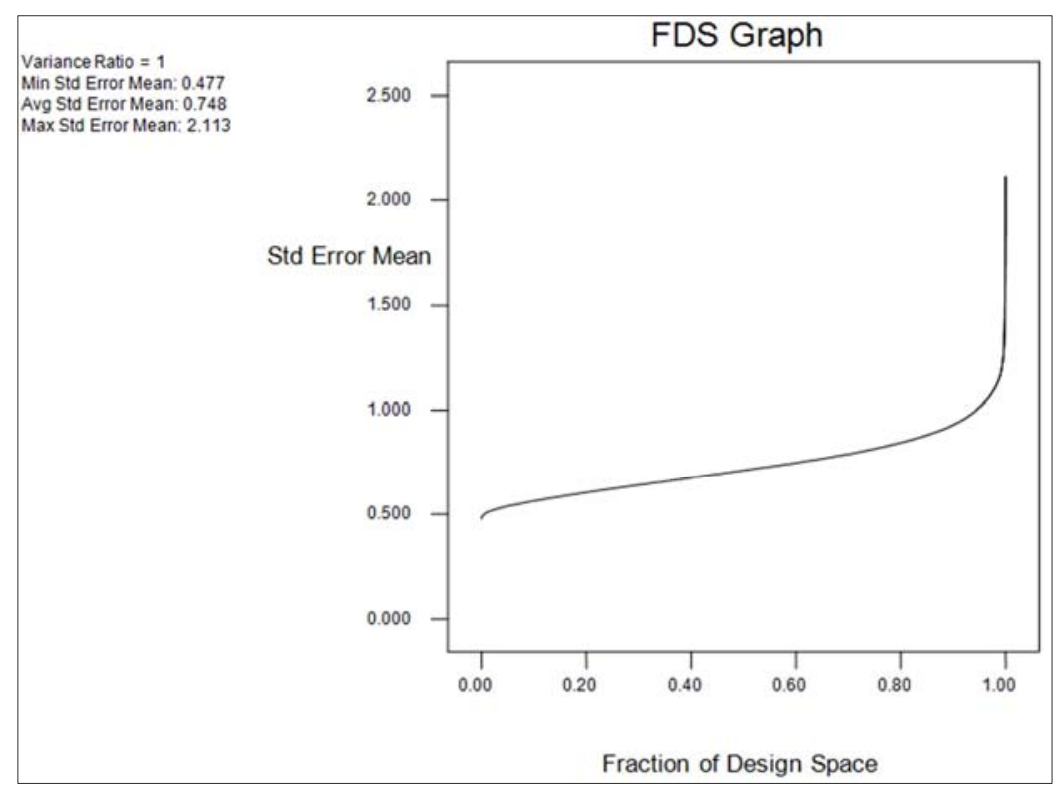

Figure 2. FDS plot for the D-optimal Split-plot Design.

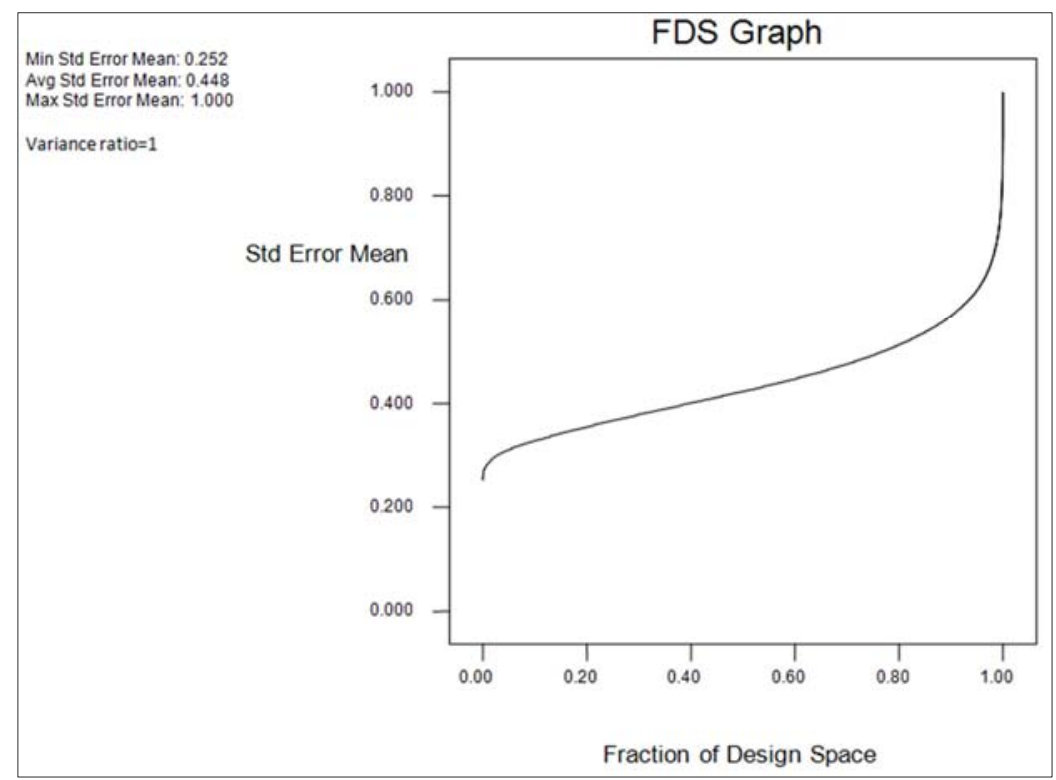

Figure 3. FDS Plot for the I-optimal Split-plot Design. 
Fig. 2 showed a relatively uniform distribution of the standard error mean across the design space but towards the boundaries, the standard error mean increased sharply to quite a high value. The range of the standard error mean spread across the design was quite wide in fig. 2, 1.636 units, compared to 0.748 units in fig. 3. In fig. 3 also, much of the volume of the design space was below the average of the standard error mean and was relatively more uniformly spread compared to fig. 2. In addition, the standard error mean values were smaller, with as low as 0.252 and the highest value one unit in the I-optimal split-plot design as opposed to the D-optimal split-plot design whose smallest value was 0.477 and stretched even to 2.113 . The standard error of the coefficient estimates gives a measure of the accuracy of predictions. The smaller the standard error value is, the better, for then the more the precise the measurement of the coefficients and the better the prediction.

Notably, FDS plots given in section (3.3.4) were more informative about the prediction capacities and precise estimation of the coefficient estimates of the design than the alphabetic criteria measures. The plots display the values of the standard error mean and hence the prediction patterns throughout the design space. They also show how precise the coefficient estimates would be measured throughout the design space as opposed to single values from the alphabetic criteria. This agrees with recommendations made by [ 3 and 15]. The FDS plots were found to be a commendable tool in design evaluation as they show the distribution of the prediction variance throughout the design space as opposed to a single value given by the usual alphabetic criteria.

\section{Summary, Conclusions and Recommendations}

\subsection{Summary of the Findings}

In section 3.1, two alternative arrangements of design points in a split-plot design were compared. In the first arrangement, seven mixture blends were set up at each of four points of the $2^{2}$ factorial design. The process factors were the whole-plots and the mixture components the subplots. In the second arrangement, the $2^{2}$ factorial design was set up at each of seven points of the simplex centroid design. This resulted in seven whole-plots and four sub-plots. The first arrangement was found to be more efficient and to give more precise parameter estimates in terms of A-, D- and Eoptimality criteria. In this arrangement, there were more subplots than whole-plots and the researcher was interested in getting more precise measurements among the mixtures. The split-plot design gave room to measure the effect of change of process factors on the different mixture blends.

In section 3.2, I- and D-optimal designs were constructed. The efficiency of the two optimal designs in terms of A-, Dand E-optimality criteria was compared and their FDS plots obtained. I-optimal split-plot design was found to be more efficient in terms of A- and E- optimality than the D-optimal split-plot design, thus implying better precision of parameter estimates. The FDS plots displayed the distribution of prediction variance throughout the design space. I-optimal split-plot design was preferred in terms of prediction since it had lower prediction values throughout the design space than the D-optimal split-plot design. Overall, the I-optimal splitplot design was preferred since it had the capacity for better prediction properties and precision in the measurement of the coefficients.

\subsection{Conclusions and Recommendations}

Split-plot design allows the researcher to model the effect of the mixture components and their process conditions simultaneously. The interaction effects of both mixture design and the factorial design can effectively be measured using this split-plot design. We thus recommend the employment of split-plot designs in experiments involving mixture formulations to measure the interaction effects of both the mixture components and the processing conditions. In cases where precision of the results is more desirable on the mixtures and also where the mixture blends are more than the sets of process conditions, the research recommends that the mixture experiment be set up at each of the points of a factorial design.

We also recommend to other researchers in the area of response surface methodology the use of Design-Expert ${ }^{\circledR}$ software in the construction of optimal designs and the fraction of design space plots. This software offers clear instructions that are easy to follow and produce reliable results. To a researcher whose interest is on prediction aspects of the system and is also keen on getting precise parameter estimates, we recommend the I-optimal split-plot design to be employed since it has low prediction variance in much of the design space and also gives reasonably optimal parameter estimates. In addition, optimal split-plot designs would help to reduce the number of runs in an experiment to only those that optimize the process.

\section{References}

[1] Anderson-Cook, C. M., Borror, C. M. \& Montgomery, D. C., "Response surface design evaluation and comparison," Journal of Statistical Planning and Inferences; 139: 629-641, 2009.

[2] Box, G. E. P. \& Hunter, J., "Multifactor experimental designs for exploring response surfaces," The Annals of Mathematical Statistics, 28: 195-241, 1957.

[3] Cho, T-Y., Mixture-process variable design experiments with control and noise variables within a split-plot structure. A PhD Dissertation, Arizona State University, 2010.

[4] Cornell, J. A., Experiments with Mixtures: designs, models and the analysis of mixture data, $3^{\text {rd }}$ Edition; New York, NY. John Wiley\& Sons, Inc, 2002.

[5] Design-Expert ${ }^{\circledR}$ Version 10 Software. Minneapolis, MN. State-Ease Inc, 2016. 
[6] Giovannitti-Jensen, A. \& Myers, R. H., "Graphical assessment of the prediction capability of response surface designs," Technometrics, 31: 159-171, 1989.

[7] Goldfarb, H. B., Anderson-Cook, C. M., Borror, C. M. \& Montgomery, D. C., "Fraction of design space plots for assessing mixture-process designs," Journal of Quality Technology, 36(2): 169-179, 2010.

[8] Goldfarb, H. B., Borror, C. M. \& Montgomery, D. C., "Mixture-process variable experiments with noise variables," Journal of Quality Technology, 35:393-405, 2003.

[9] Goos, P., The optimal design of blocked and split-plot experiments, New York: Springer -Verlang, 2002.

[10] Kowalski, S. M., Cornell, J. A., \& Vining, G. G., "Split-Plot Designs and estimation methods for mixture experiments with process variables," Technometrics, 44 (1): 72-79, 2002.

[11] Liang, J. D., Myers, R. A. \& Robinson, T. J., "Fraction of
Design Space Plots for Split-Plot Designs," Quality and Reliability Engineering International, 22: 275-289, 2006.

[12] Lin, L-Y., Robust split-plot designs for model misspecification. Department of Applied Mathematics and Institute of Statistics, National Chung Hsing University, Taichung, Taiwan, 40227, 2016.

[13] Montgomery, D. C., Design and Analysis of Experiments, New York, John Wiley \& Sons, Inc.2009.

[14] Schoonees, P., Niel le Roux, N. J. \& Coetzer, R. L. J., "Flexible Graphical Assessment of Experimental Designs in R: The vdg package," Journal of Statistical Software, 74(3), 2016.

[15] Zahran, A., Anderson-Cook, C. M. \& Myers, R. H., "Fraction of Design Space to Assess Prediction Capability of Response Surface Designs," Journal of Quality Technology, 35: 377386, 2003. 